\title{
High prevalence of subclinical tuberculosis in HIV-1-infected persons without advanced immunodeficiency: implications for TB screening
}

\author{
Tolu Oni, ${ }^{1,2}$ Rachael Burke, ${ }^{3}$ Relebohile Tsekela, ${ }^{1}$ Nonzwakazi Bangani, ${ }^{1}$ \\ Ronnett Seldon, ${ }^{1}$ Hannah P Gideon, ${ }^{1}$ Kathryn Wood, ${ }^{1}$ Katalin A Wilkinson, ${ }^{1,4}$ \\ Tom H M Ottenhoff, ${ }^{5}$ Robert J Wilkinson ${ }^{1,2,4}$
}

\begin{abstract}
- Additional materials are published online only. To view these files please visit the journal online (http://thorax.bmj. com)

${ }^{1}$ Institute of Infectious Diseases and Molecular Medicine, Faculty of Health Sciences, University of Cape Town, Observatory, South Africa

${ }^{2}$ Division of Medicine, Imperial College London, London, UK ${ }^{3}$ Department of Public Health, University of Oxford, Old Road Campus, Headington, Oxford, UK

${ }^{4}$ Medical Research Council, National Institute for Medical Research, Mill Hill, London, UK ${ }^{5}$ Leiden University Medical Center, Leiden, The Netherlands
\end{abstract}

\section{Correspondence to}

Tolu Oni, 3.03 Wolfson Pavillion, Institute of Infectious Diseases and Molecular Medicine, Faculty of Health Sciences, University of Cape Town, Observatory 7925

South Africa;

tolullahoni@doctors.org.uk

Received 24 January 2011 Accepted 28 April 2011 Published Online First 31 May 2011

\section{ABSTRACT}

Background The prevalence of asymptomatic tuberculosis (TB) in recently diagnosed HIV-1-infected persons attending pre-antiretroviral therapy (ART) clinics is not well described. In addition, it is unclear if the detection of Mycobacterium tuberculosis in these patients clearly represents an early asymptomatic phase leading to progressive disease or transient excretion of bacilli.

Objective To describe the prevalence and outcome of subclinical TB disease in HIV-1-infected persons not eligible for ART

Methods The study was conducted in 274 asymptomatic ART-naïve HIV-1-infected persons in Khayelitsha Day Hospital, Cape Town, South Africa. All participants were screened for TB using a symptom screen and spoligotyping was performed to determine genotypes.

Results The prevalence of subclinical TB disease was $8.5 \%(95 \% \mathrm{Cl} 5.1 \%$ to $13.0 \%)(\mathrm{n}=18$; median days to culture positivity 17 days), with $22 \%$ of patients being smear-positive. Spoligotyping showed a diverse variety of genotypes with all paired isolates being of the same spoligotype, effectively excluding cross-contamination. $56 \%$ of patients followed up developed symptoms 3 days to 2 months later. All were well and still in care 6-12 months after TB diagnosis; $60 \%$ were started on ART. A positive tuberculin skin test (OR 4.96, $p=0.064$ ), low CD4 count (OR 0.996, $p=0.06$ ) and number of years since HIV diagnosis (OR 1.006, $p=0.056$ ) showed trends towards predicting TB disease.

Conclusion This study found a high prevalence but good outcome (retained in care) of subclinical TB disease in HIV1-infected persons. The results suggest that, in high HIV/ TB endemic settings, a positive HIV-1 test should prompt TB screening by sputum culture irrespective of symptoms, particularly in those with a positive tuberculin skin test, longer history of HIV infection and low CD4 count. Operational difficulties in resource-constrained settings with respect to screening with TB culture highlight the need for rapid and affordable point-of-care tests to identify persons with clinical and subclinical TB disease.

\section{INTRODUCTION}

Tuberculosis (TB) is the most common opportunistic infection and is the leading cause of mortality in HIV-1-infected persons. ${ }^{1}$ Reducing the morbidity and mortality of $\mathrm{TB}$ in HIV-1 co-infected persons requires an improved understanding of the epidemiology of $\mathrm{TB}$, including the prevalence of

\section{Key messages}

What is the key question?

- How prevalent is subclinical culture-positive TB and what are the clinical outcomes in HIV-1. infected adults without advanced immunodeficiency in a high HIV/TB burden setting?

What is the bottom line?

- It is not clear what the public health implications are of the detection of Mycoplasma tuberculosis in asymptomatic patients and whether this represents a clinically relevant disease state.

Why read on?

- We describe the prevalence and clinical outcomes of subclinical TB in HIV co-infected adults, explore potential predictors of TB in asymptomatic persons and make policy recommendations for TB screening in high transmission settings.

and risk factors for latent $\mathrm{TB}$ infection and $\mathrm{TB}$ disease in this population.

South Africa has a very high incidence of $T B$ with an estimated national incidence rate of 948/ 100000 population per year in 2007. Against this background of high prevalence, short course directly observed therapy, which is predominantly dependent on passive case finding, has been implemented with considerable success. However, this passive approach has some recognised weaknesses. Notably, it assumes that persons with TB will have symptoms as a trigger to seek investigation for TB. However, waiting for symptomatic persons to present for $\mathrm{TB}$ screening can result in a delay in investigation for $\mathrm{TB}$ and consequently continued transmission in the community as well as a potentially worse outcome

$\mathrm{TB}$ infection is considered to lead to one of three outcomes: $5 \%$ are thought to progress to active $\mathrm{TB}$ disease (primary TB) while the majority develop an acquired immune response but no signs or symptoms of disease (latent TB infection). Among those with latent infection there is a $10 \%$ risk per lifetime (up to $10 \%$ risk per year in HIV-1-infected persons) of progressing to active TB (post-primary TB). It has recently been proposed that the latent $\mathrm{TB}$ infection phase, rather than being static, comprises a more dynamic spectrum of responses to $\mathrm{TB}$ 
infection that includes innate immune, acquired immune, quiescent infection, active infection or clinical disease. ${ }^{2}$ Of note, this spectrum predicts the existence of a minimally symptomatic phase of infection during which patients nevertheless become infectious.

\section{METHODS}

\section{Study location and design}

The study was conducted at site B Day Hospital, a primary care hospital in the informal township of Khayelitsha. Located on the outskirts of Cape Town, Khayelitsha has a population of over 400000 and a TB case notification rate of over 1600/ $100000,70 \%$ of which are associated with HIV-1. The study was conducted as part of a larger cross-sectional TB diagnostics study.

\section{Participants}

Study participants were recruited from the HIV pre-antiretroviral therapy (ART) clinic between February 2008 and March 2010. Written informed consent was obtained from all participants and the University of Cape Town research ethics committee approved the study (REC 012/2007).

Two hundred and seventy-four asymptomatic ART-naïve HIV-1-infected persons were recruited. At recruitment all participants were screened for TB and a baseline CD4 count was performed. All participants received the tuberculin skin test (TST) using 2 TU tuberculin PPD RT23 injected intradermally into the volar aspect of the forearm. All those with a skin induration diameter of $\geq 5 \mathrm{~mm}$ had a chest $\mathrm{x}$-ray performed and were started on isoniazid preventive therapy for 6 months if the $\mathrm{x}$-ray was normal. For comparison, $162 \mathrm{HIV}-1$-infected patients with TB symptoms diagnosed with culture-positive TB were recruited from the $\mathrm{TB}$ clinic in the same setting.

\section{TB screening}

All participants were screened for TB using a symptom screen (cough of any duration, fever, unintentional weight loss, loss of appetite and drenching night sweats) and study-specific induced sputum samples were collected. Microscopy and culture were performed on the collected specimens and processed at a national reference laboratory in Cape Town. A TB case was defined as one with at least one culture-positive sputum specimen in the presence (symptomatic TB cases) or absence (asymptomatic subclinical TB cases) of symptoms.

\section{Spoligotyping}

Genomic DNA was prepared as previously described. ${ }^{3}$ Twentysix heat-killed isolates were prepared from MGIT cultures and two heat-killed isolates were prepared from Lowenstein-Jensen slope cultures. The DNA was used to perform spoligotyping as previously described ${ }^{4}$ using a commercially available kit from Ocimum Biosolutions BV, The Netherlands. The hybridisation patterns obtained were used to assign the isolates to genotype families using the SPOTCLUST SpolDB3 database. ${ }^{5}$

\section{Statistical methods}

Baseline characteristics were tabulated and compared between groups using the $\chi^{2}$ test for categorical variables and Wilcoxon test for continuous variables that were not normally distributed. Predictors of disease status were analysed by logistic regression. The model was built both manually and using the stepwise method including variables with a $\mathrm{p}$ value $<0.10$. Subsequent inclusion of other variables in turn did not improve the fit of the model to the data (using the Pearson $\chi^{2}$ goodness-of-fit test) and these variables were therefore excluded. Median CD4 counts were compared across the symptomatic $\mathrm{TB}$, asymptomatic $\mathrm{TB}$ and asymptomatic non-TB groups using the Kruskal-Wallis test. All $p$ values were two-sided and $p$ values $<0.05$ were considered statistically significant. All data were analysed using Stata 10.0 (StataCorp) and GraphPad Prism V.5.0 software.

\section{RESULTS}

\section{Prevalence of asymptomatic culture-positive TB}

Of the 274 asymptomatic HIV-1-infected persons recruited sputum samples were obtained from 213 (figure 1). Overall, 18 (8.5\%, 95\% CI 5.1\% to $13.0 \%$ ) were diagnosed with TB disease. A summary of the characteristics and outcomes of these patients is shown in the online supplement. Seventeen were found to be TB culture-positive on at least one specimen (median days to culture positivity 17 days), with three having an abnormal x-ray. Four of the 18 (22\%) were sputum smear microscopy positive. One patient was diagnosed with TB solely on the basis of an $\mathrm{x}$-ray reviewed by two doctors at the TB clinic while one person was diagnosed with culture-positive TB isolated from a cold abscess of the elbow. Four patients had negative TST results and therefore did not have an x-ray performed. These patients had moderate to severe immunosuppression with CD4 counts ranging from 11 to 250 . Of the 14 patients in whom an $x$-ray was performed, 10 patients $(71 \%)$ had normal x-rays. Face-toface interviews were conducted with 11 of the 18 patients and folder reviews were undertaken of a further five patients 1 year after TB diagnosis. Although all participants had no TB symptoms at baseline, 9 of the 16 (56\%) developed symptoms 3 days to 2 months later (median 28 days after diagnosis), including one

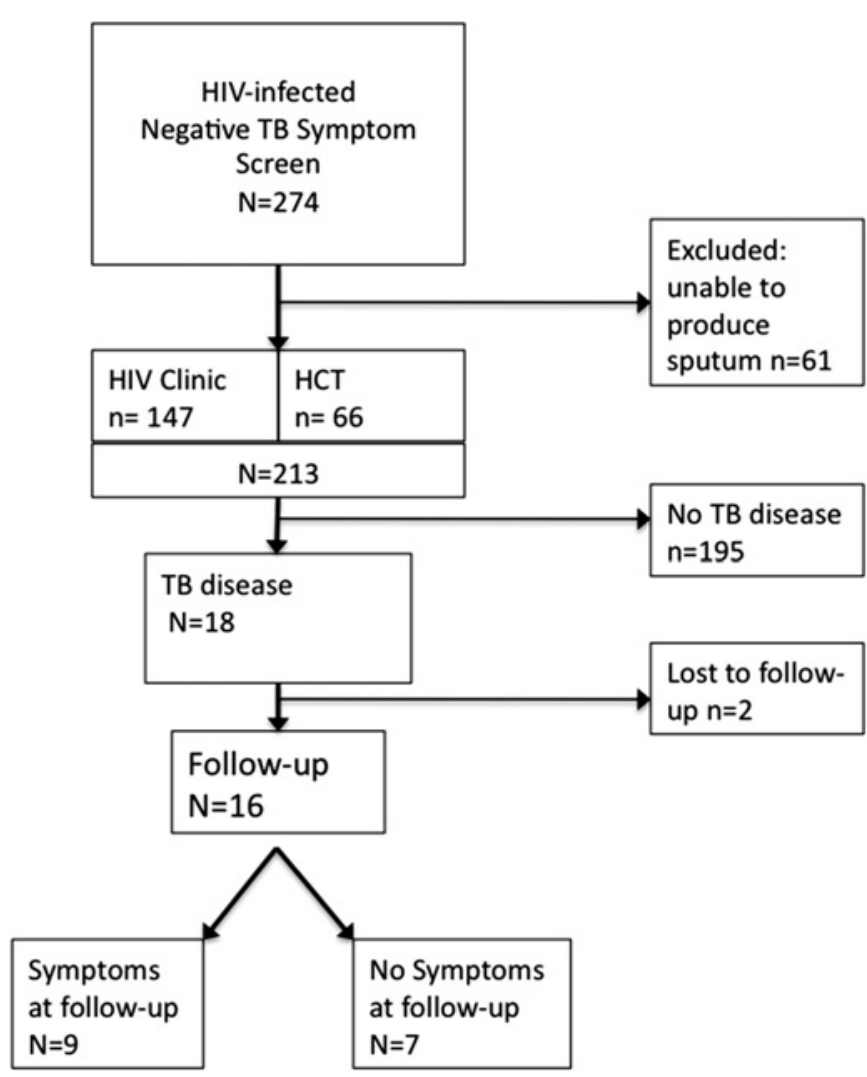

Figure 1 Recruitment flow diagram of participants into the study. HCT, HIV counselling and testing. 
patient who was admitted to hospital with disseminated TB 10 days after recruitment (patient 1 in table 1 in the online supplement). Seven of the 16 received isoniazid preventive therapy for up to 3 months. There was no difference in the proportion that developed symptoms between those who did and did not receive isoniazid preventive therapy (57\% vs $56 \%$ ). All 16 were started on and completed TB treatment irrespective of whether symptoms subsequently developed and were still in care 6-12 months after TB diagnosis and treatment; $60 \%$ became eligible for and were started on ART in accordance with South African national guidelines.

At baseline a greater proportion of the asymptomatic TB group had a TST $\geq 5 \mathrm{~mm}$ than those with no TB (78\% vs $56 \%$; $\mathrm{p}=0.051)$ and a higher proportion of asymptomatic TB patients had CD4 counts $\leq 200$ compared with the non-TB group $(p=0.055)$. There was no statistically significant difference between the two groups at baseline with respect to all other characteristics measured (table 1).

Having found a high prevalence of asymptomatic disease, we wished to exclude cross-contamination as a possible cause of culture-positive results using spoligotyping. We found a diverse variety of genotypes (see table 1 in the online supplement), with all paired isolates being of the same spoligotype, thereby effectively excluding cross-contamination as a source of culturepositive results.

Table 1 Baseline characteristics by TB disease group

\begin{tabular}{|c|c|c|c|c|}
\hline & $\begin{array}{l}\text { Non-TB } \\
\text { n }(\%)\end{array}$ & $\begin{array}{l}\text { Asymptomatic TB } \\
\text { n (\%) }\end{array}$ & p Value & $\begin{array}{l}\text { Overall } \\
\text { N (\%) }\end{array}$ \\
\hline \multicolumn{5}{|l|}{ Sex } \\
\hline Female & $200(80)$ & $15(83)$ & 0.732 & $215(80)$ \\
\hline Male & $50(20)$ & $3(17)$ & & $53(20)$ \\
\hline \multicolumn{5}{|l|}{ Previous TB } \\
\hline No & $193(85)$ & $13(75)$ & 0.286 & $205(84)$ \\
\hline Yes & $34(15)$ & $4(25)$ & & $38(16)$ \\
\hline \multicolumn{5}{|l|}{ BCG scar } \\
\hline No & $109(54)$ & $10(59)$ & 0.699 & $119(54)$ \\
\hline Yes & $93(46)$ & $7(41)$ & & $100(46)$ \\
\hline \multicolumn{5}{|l|}{ TB contact } \\
\hline No & $176(80)$ & $13(76)$ & 0.756 & $189(79)$ \\
\hline Yes & $45(20)$ & $4(24)$ & & $49(21)$ \\
\hline \multicolumn{5}{|l|}{ CD4 count } \\
\hline$<200$ & $45(20)$ & $7(39)$ & 0.055 & $52(21)$ \\
\hline$\geq 200$ & $183(80)$ & $11(61)$ & & $194(79)$ \\
\hline \multicolumn{5}{|l|}{ Tuberculin skin test } \\
\hline$<5 \mathrm{~mm}$ & $98(44)$ & $4(22)$ & 0.051 & $102(42)$ \\
\hline$\geq 5 \mathrm{~mm}$ & $124(56)$ & $14(78)$ & & $138(58)$ \\
\hline \multicolumn{5}{|l|}{ Smoker } \\
\hline No & $164(81)$ & $12(75)$ & 0.546 & $176(81)$ \\
\hline Yes & 38 (19) & $4(25)$ & & $42(19)$ \\
\hline \multicolumn{5}{|l|}{ Alcohol consumption } \\
\hline No & $159(79)$ & $11(65)$ & 0.183 & $170(78)$ \\
\hline Yes & $43(21)$ & $6(35)$ & & $49(22)$ \\
\hline \multicolumn{5}{|l|}{ Time in Khayelitsha } \\
\hline$<6$ months & $5(3)$ & $0(0)$ & 0.212 & $5(2)$ \\
\hline $6-12$ months & $6(3)$ & $2(12.5)$ & & $8(4)$ \\
\hline $1-5$ years & $43(22)$ & $2(12.5)$ & & $45(21)$ \\
\hline$>5$ years & $140(72)$ & $12(75)$ & & $152(73)$ \\
\hline \multicolumn{5}{|l|}{ Medians (IOR) } \\
\hline Age & $30(27-37)$ & $34(27-38)$ & 0.365 & 31 \\
\hline CD4 cell count $/ \mathrm{mm}^{3}$ & $322(225-446)$ & $249(170-332)$ & 0.026 & 312 \\
\hline Body mass index & $26(22-31)$ & $24(23-27)$ & 0.140 & 26 \\
\hline $\begin{array}{l}\text { Days since HIV-1 } \\
\text { diagnosis }\end{array}$ & $319(17-1118)$ & $640(20-1389)$ & 0.313 & 325 \\
\hline
\end{tabular}

\section{Comparison with symptomatic TB cases}

We examined the CD4 count distribution, proportion of smearnegative culture-positive patients and days to culture positivity in asymptomatic TB cases and compared them with symptomatic TB cases and a control group of asymptomatic HIV-1infected persons with no TB. Patients in all three groups came from the same setting, had similar baseline characteristics and were recruited under the same conditions. CD4 counts were highest in those with no TB (median 322, IOR 225-446) and lowest in symptomatic TB patients (median 148, IOR 64-259), with the median CD4 count of asymptomatic TB cases being between them (median 249, IOR 170-332; table 2). This trend to a decline in CD4 count was statistically significant $(p<0.001)$. In addition, there was a significant difference in the median days to culture positivity in the symptomatic and asymptomatic TB groups with a median of 17 days (IOR 12-39) in those with a negative TB screen and 7 days (IOR 6-12) in symptomatic TB cases $(p<0.001$, figure 2$)$. Seventy per cent of asymptomatic TB patients had smear-negative TB compared with only $16 \%$ of symptomatic TB patients $(p<0.001)$, suggesting that the bacterial burden is lower in patients with subclinical $T B$.

\section{Risk factors for asymptomatic TB disease}

Of the asymptomatic HIV-1-infected persons recruited, 69\% were recruited from the HIV pre-ART clinic and $31 \%$ from the HIV counselling and testing clinic (HCT) within 30 days of HIV-1 diagnosis. There was no significant difference in the prevalence of $\mathrm{TB}$ disease between persons recruited from the two clinics. In addition, there was no significant difference in CD4 counts $(p=0.600)$ between persons recruited from the HCT clinic (median CD4 count 326 (IOR 166-456); median time since HIV-1 diagnosis 6 days (IOR 0-13)) and those from the HIV-1 clinic (median CD4 count 305 (IOR 230-433); median time since HIV-1 diagnosis 776 days (IOR 278-1585)). These two groups did not differ significantly in other baseline characteristics measured.

To further explore the risk factors for asymptomatic TB disease, we conducted multivariable logistic regression on all asymptomatic persons with the presence or absence of $\mathrm{TB}$ disease as an outcome variable (table 3 ). A positive TST (OR 4.96, $\mathrm{p}=0.064$ ), a longer history of HIV infection (OR 1.006, $\mathrm{p}=0.056$ ) and a low CD4 count (OR 0.996, $\mathrm{p}=0.060$ ) showed trends (albeit not statistically significant) towards being predictive of subclinical TB disease.

\section{DISCUSSION}

We report an $8.5 \%$ prevalence of asymptomatic TB disease among HIV-1-infected persons not receiving ART in Cape Town. We also showed that most of these cases progressed clinically with development of symptoms a median of 28 days after diagnosis, as well as good adherence and treatment outcome at follow-up 6-12 months after TB diagnosis. This suggests an increasing bacterial load after initial screening and that screening before the likely development of symptoms was beneficial in indicating the presence of actively replicating bacilli. This early

Table 2 CD4 count gradient across TB disease groups

\begin{tabular}{llll}
\hline & CD4 count & IOR & $\begin{array}{l}\text { p Value } \\
\text { (Kruskal-Wallis) }\end{array}$ \\
\hline Asymptomatic, no TB disease & 322 & $225-446$ & $<0.0001$ \\
Asymptomatic, TB disease & 249 & $170-332$ & \\
Symptomatic, TB disease & 148 & $64-259$ & \\
\hline
\end{tabular}




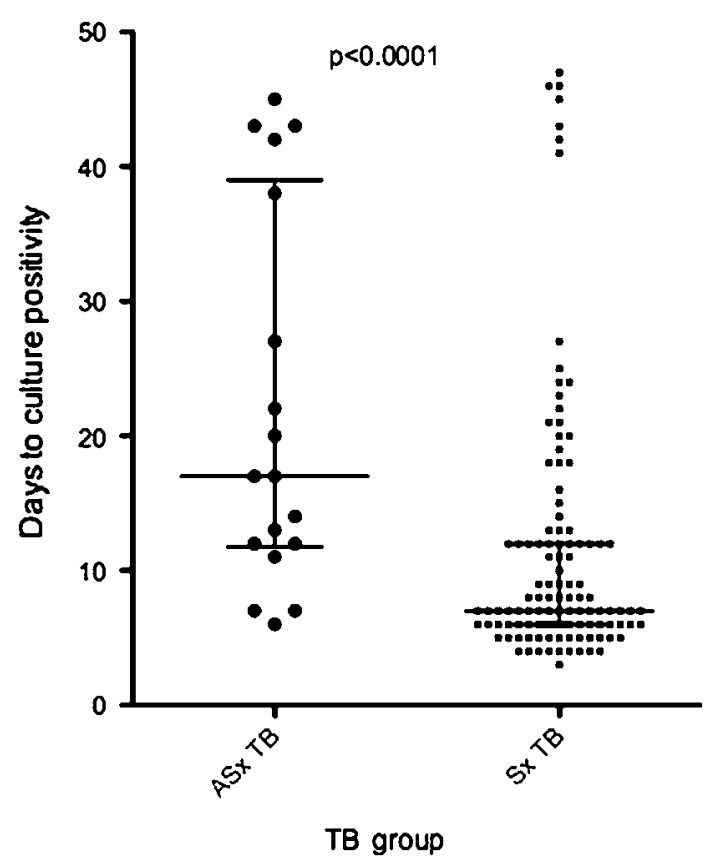

Figure 2 Scatterplot showing a comparison of number of days to culture positivity in symptomatic and asymptomatic TB cases. ASx, asymptomatic subclinical TB cases; $\mathrm{Sx}$, symptomatic TB cases.

diagnosis resulted in patients receiving treatment for TB earlier than would otherwise have occurred. This finding has potential significance for TB control programmes implemented in this setting, in addition to wider public health implications.

Our findings support the hypothesis that TB infection presents as a spectrum from innate immune clearance to clinical disease and development of symptoms. ${ }^{2}$ In a previous study we demonstrated a significant correlation between bacterial load (measured by degree of smear positivity in TB patients) and the interferon gamma release assay (IGRA) response, as well as a trend towards a correlation between the IGRA response and days to culture positivity. ${ }^{6}$ This suggested an association between bacterial load and days to culture positivity. Using days to culture positivity as a marker of bacterial load, our result showing a longer period to culture positivity in asymptomatic than in symptomatic persons suggests a higher bacterial load in symptomatic persons than in asymptomatic persons. Our finding of a greater proportion of smear-negative TB cases in the asymptomatic cases than in the symptomatic TB cases $(70 \%$ vs
$16 \%$ ) also supports this interpretation. In addition, by comparing asymptomatic persons with no $\mathrm{TB}$, asymptomatic cases with TB and symptomatic TB cases, we demonstrate a spectrum in CD4 count distribution with decreasing CD4 counts across these three groups.

Several studies have reported subclinical asymptomatic TB disease as defined by culture-positive $\mathrm{TB}$ in the absence of $\mathrm{TB}$ symptoms. Swaminathan et al screened HIV-1-infected persons in India and reported a $4 \%$ prevalence of unrecognised $\mathrm{TB}$ in HIV-1-infected persons with minimal or no symptoms, normal chest $\mathrm{x}$-rays, negative smears and different levels of immunosuppression. ${ }^{7}$ Mtei et al recruited HIV-1-infected persons with CD4 counts $>200 / \mathrm{mm}^{3}$ and found a $15 \%$ prevalence of TB, $29 \%$ of which was subclinical. ${ }^{8}$ Patients with subclinical TB had a better prognosis than those with clinical disease, with no clinical predictors of subclinical TB identified. In addition, an intensified TB case-finding study by Shah et al on newly diagnosed HIV-1-infected persons in Ethiopia found that $16 \%$ of all prevalent TB cases were asymptomatic. ${ }^{9}$ These studies highlight the entity that is subclinical TB. A TB prevalence survey in Zimbabwe found that $41 \%$ of all prevalent cases were subclinical at the time of screening, $82 \%$ of whom had developed symptoms within a few weeks of follow-up; no significant risk factors for prevalent culture-positive disease were found. ${ }^{10}$ One theory suggested is that, in a healthcare system with a strong TB diagnosis and treatment programme, severe cases are identified early leaving TB patients with fewer clinical symptoms to be identified through provider-initiated TB screening. ${ }^{11}$ Results from a meta-analysis of yield of HIV-1-associated TB during intensified case-finding suggested that $\mathrm{TB}$ cases diagnosed by active screening might be less likely to adhere to treatment, resulting in poor outcomes. ${ }^{12}$ In our study, all patients started on TB treatment completed the 6-month course with good outcomes and remained in care at 6-12 months after treatment.

We also explored potential risk factors for $\mathrm{TB}$ disease in asymptomatic HIV-1-infected persons. We found a trend towards a TST $\geq 5 \mathrm{~mm}$, lower CD4 count and increasing number of days since HIV-1 diagnosis being associated with an increased risk of $\mathrm{TB}$ disease. There was a $5 \%$ decrease in $\mathrm{TB}$ disease risk for every increase in the CD4 count of 10 cells $/ \mathrm{mm}^{3}$, and an increase of $24 \%$ in the risk of TB disease for every year after HIV-1 diagnosis. This raises concern about the possibility of the association we report being linked to nosocomial transmission of TB. However, when we compared the prevalence of asymptomatic $\mathrm{TB}$ in those who had been in HIV care for $<30$ days with those who had received a diagnosis of HIV-1

Table 3 Risk factors for asymptomatic TB disease

\begin{tabular}{|c|c|c|c|c|}
\hline & \multicolumn{2}{|c|}{ Univariate analysis } & \multicolumn{2}{|l|}{ Multivariable analysis } \\
\hline & $\overline{\mathbf{O R}}$ & $\overline{p \text { Value }}$ & $\overline{\mathrm{OR}(95 \% \mathrm{CI})}$ & $\overline{p \text { Value }}$ \\
\hline CD4 cell count $/ \mathrm{mm}^{3}$ & 0.996 & 0.035 & $0.996(0.992$ to 1.000$)$ & 0.060 \\
\hline Tuberculin skin test $\geq 5 \mathrm{~mm}$ & 2.77 & 0.081 & $4.96(0.990$ to 17.662$)$ & 0.064 \\
\hline Days since HIV-1 diagnosis & 1.00 & 0.095 & $1.006(0.999$ to 1.010$)$ & 0.056 \\
\hline Age (years) & 1.02 & 0.496 & & \\
\hline Male sex & 0.80 & 0.732 & & \\
\hline Body mass index & 0.92 & 0.112 & & \\
\hline History of previous TB & 1.89 & 0.293 & & \\
\hline Smoker & 1.44 & 0.548 & & \\
\hline BCG scar presence & 0.82 & 0.699 & & \\
\hline Contact with TB & 1.20 & 0.756 & & \\
\hline Alcohol consumption & 2.02 & 0.190 & & \\
\hline Time in Khayelitsha $>5$ years & 1.16 & 0.808 & & \\
\hline
\end{tabular}


$\geq 30$ days earlier and were established in the pre-ART clinic, there was no statistically significant difference in the prevalence of subclinical TB.

The question of the public health relevance of asymptomatic persons found to be TB culture-positive with respect to the contribution to transmission is potentially important. It has been shown that smear-negative culture-positive TB is transmissible but less infectious than smear-positive disease, with smear-negative disease being responsible for up to $17 \%$ of transmitted $\mathrm{TB}^{13}$ In our study, $22 \%$ of asymptomatic cases were smear positive and therefore more infectious; $56 \%$ progressed to develop symptoms within 2 months, suggesting that they would ultimately have been likely to contribute to transmission.

\section{Limitations of the study}

The specificity of culture can be affected by contamination as a result of laboratory manipulations. We excluded crosscontamination as a cause of culture-positive results by strain typing of isolates. In addition, the reference laboratory where the samples were processed has previously reported low contamination rates; of 500 dummy sputum samples, none were false positive for Mycobacterium tuberculosis.

\section{CONCLUSION}

Our results show a high prevalence of asymptomatic TB disease in HIV-1 co-infected persons in Cape Town. These results suggest that, in high HIV/TB prevalence settings, a diagnosis of HIV-1 should prompt a TB test by sputum culture even in the absence of symptoms, particularly in persons with positive TST reactions, lower $\mathrm{CD} 4$ counts and a longer history of HIV. The WHO guidelines for isoniazid preventive therapy in resourceconstrained settings recommend that all HIV-infected persons, irrespective of the degree of immunosuppression, should be screened for TB and isoniazid preventive therapy provided if no symptoms. ${ }^{14}$ A recent meta-analysis by Getahun et al that aimed to develop a standardised TB screening tool for HIV-infected persons found that the clinical rule of any of the following symptoms (current cough, night sweats, fever or weight loss) excluded active TB disease with a sensitivity of $78.9 \%$ and specificity of $49.6 \%$. ${ }^{15}$ However, it was noted that the negative predictive value of this screening tool was dependent on the prevalence of $\mathrm{TB}$ in the screened population, ranging from $97.7 \%$ at a TB prevalence of $5 \%$ to $90 \%$ at a TB prevalence of $20 \%$. Isoniazid preventive therapy has been shown to significantly decrease the incidence of TB in HIV-infected persons, but is not widely implemented owing to reservations about effective exclusion of active TB. Therefore, in most high-burden lowresource settings, Getahun et al show that this clinical tool will be sufficient to exclude active $\mathrm{TB}$, permitting implementation of isoniazid preventive therapy. However, in settings like the Western Cape of South Africa with one of the most intense TB transmission rates in the world, our results show that this clinical tool will miss $8.5 \%$ of HIV-infected persons with subclinical TB.

However, we acknowledge the operational difficulties in the majority of resource-constrained settings with respect to screening with TB culture, highlighting the need for new rapid and affordable point-of-care diagnostic tests to identify persons with clinical and subclinical TB disease.

Acknowledgements The authors thank Kees Franken for producing the fusion protein and Dr Virginia de Azevedo, Khayelitsha sub-district manager, for facilitating the study.

Funding RJW is funded by the Wellcome Trust $(084323,088316), \mathrm{MRC}$ (UK) and the Department of Health, South Africa. KAW is funded by MRC (UK). This study was supported by the ILULU Consortium which is funded by a grant from the European Union (Sante/2006/105-061)and Bill and Melinda Gates Foundation GC\#6-74 37772 The funders did not play a role in the design of the study or preparation of the manuscript.

\section{Competing interests None.}

Ethics approval This study was conducted with the approval of the University of Cape Town.

Contributors TO contributed to data collection and was responsible for data analysis and writing of the manuscript. RB contributed to data collection and analysis. RT contributed to data collection. RS was responsible for strain typing of culture isolates $\mathrm{NB}, \mathrm{HPG}$ and KW were responsible for laboratory processing of specimens. KAW and THMO contributed to the writing of the manuscript. THMO supervised the preparation of the recombinant fusion protein used in the IGRA. RJW contributed to study design and writing of the manuscript.

Provenance and peer review Not commissioned; externally peer reviewed.

\section{REFERENCES}

1. Sterling TR, Pham PA, Chaisson RE. HIV infection-related tuberculosis: clinical manifestations and treatment. Clin Infect Dis 2010;50(Suppl 3):S223-30.

2. Young DB, Gideon HP, Wilkinson RJ. Eliminating latent tuberculosis. Trends Microbiol 2009;17:183-8.

3. Evans J, Stead MC, Nicol MP, et al. Rapid genotypic assays to identify drugresistant Mycobacterium tuberculosis in South Africa. J Antimicrob Chemother 2009;63:11-16.

4. Kamerbeek J, Schouls L, Kolk A, et al. Simultaneous detection and strain differentiation of Mycobacterium tuberculosis for diagnosis and epidemiology. J Clin Microbiol 1997;35:907-14.

5. Ani A, Bruvik T, Okoh Y, et al. Genetic diversity of Mycobacterium tuberculosis complex in Jos, Nigeria. BMC Infect Dis 2010;10:189.

6. Oni T, Patel J, Gideon HP, et al. Enhanced diagnosis of HIV-1 associated tuberculosis by relating T-SPOT.TB and CD4 counts. Eur Respir J 2010;36:594-600.

7. Swaminathan S, Paramasivan CN, Kumar SR, et al. Unrecognised tuberculosis in HIV-infected patients: sputum culture is a useful tool. Int J Tuberc Lung Dis 2004;8:896-8.

8. Mtei L, Matee M, Herfort 0 , et al. High rates of clinical and subclinical tuberculosis among HIV-infected ambulatory subjects in Tanzania. Clin Infect Dis 2005; 40:1500-7.

9. Shah S, Demissie M, Lambert L, et al. Intensified tuberculosis case finding among HIV-Infected persons from a voluntary counseling and testing center in Addis Ababa, Ethiopia. J Acquir Immune Defic Syndr 2009;50:537-45.

10. Corbett EL, Bandason T, Cheung YB, et al. Epidemiology of tuberculosis in a high HIV prevalence population provided with enhanced diagnosis of symptomatic disease. PLoS Med 2007; 4:e22

11. Corbett EL, Zezai A, Cheung YB, et al. Provider-initiated symptom screening for tuberculosis in Zimbabwe: diagnostic value and the effect of HIV status. Bull World Health Org 2010;88:13-21.

12. Kranzer K, Houben RM, Glynn JR, et al. Yield of HIV-associated tuberculosis during intensified case finding in resource-limited settings: a systematic review and metaanalysis. Lancet Infect Dis 2010;10:93-102.

13. Behr M, Warren S, Salamon H, et al. Transmission of Mycobacterium tuberculosis from patients smear-negative for acid-fast bacilli. Lancet 1999;353:444-9.

14. World Health Organisation. Guidelines For Intensified Tuberculosis Case-Finding and Isoniazid Preventive Therapy for People Living with HIV in Resource- Constrained Settings. Geneva, Switzerland: World Health Organisation. 2011:1-50.

15. Getahun HKW, Heilig CM, Corbett EL, et al. Development of a standardized screening rule for tuberculosis in people living with HIV in resource-constrained settings: individual participant data meta-analysis of observational studies. PLoS Med 2011;8:1-14. 